\title{
CURVED FLATS IN SYMMETRIC SPACES
}

\author{
DIRK FERUS AND FRANZ PEDIT
}

\section{INTRODUCTION}

Over the past years many problems in classical surface theory and also more generally, submanifold theory, have been linked to certain types of completely integrable non-linear PDE (soliton equations). In certain cases this has already been known to geometers in the last century but the explicit construction of solutions by the finite gap integration scheme has only been achieved recently. The crucial ingredient is to rewrite the equations at hand as zero-curvature equations involving a spectral parameter — usually given by a non-trivial geometrical deformation of the surface. The finite gap theory then provides special solutions to those zero-curvature equations parameterized by certain algebraic curves, the spectral curves, which in certain cases account for all solutions. A standard example is that of (pluri) harmonic maps into symmetric spaces [3] which contains many examples of surface theory - constant mean and Gauss curvature surfaces, Willmore surfaces, minimal immersions - as special cases. Recently also the isometric immersion equations for maps between space forms (with flat normal bundle) [4], conformally flat 3-folds in the 4-sphere [8, 9] and isothermic surfaces [2] have been shown to fit into this approach.

In this paper we introduce a natural class of maps into symmetric spaces, curved flats, which, in a certain sense, contains all the above examples as special cases. A curved flat is simply a map into a symmetric space which is tangent at each point to a flat of the symmetric space, i.e. each tangent space is abelian. Since curved flats are intrinsically flat (Theorem 2) they may also be viewed as natural analogues of developable surfaces in 3-space.

Important examples of curved flats arise from isometric immersions of space forms into space forms via their Gauss maps (Section 3). The developing isometry identifies (locally) the curved flat with a Cartan subalgebra of the Grassmanian (in which the Gauss map takes values in). The roots of the Cartan subalgebra are mapped via this isometry into principal curvature coordinates of the corresponding isometric immersion (Section 4). Further examples of curved flats come from isothermic surfaces [2] and conformally flat 3-folds in the 4-sphere [8, 9]. Of course, every curve in a symmetric space is a curved flat.

Besides their geometric relevance curved flats are also interesting from the integrable systems point of view: scaling the derivative of a curved flat gives rise to a non-trivial deformation which obviously preserves the curved flat condition (Section 5). Therefore one can write the curved flat condition as a zero-curvature equation on a Lie algebra valued 1-form involving the deformation - spectral-parameter linearly. Introducing the necessary loop algebras in Section 6 we then apply the finite type integration scheme to construct solutions to the curved flat equations from a hierarchy of commuting algebraically integrable ODE in Lax form. This gives a space of solutions which has the functional dimension of finitely many functions of a single variable. At least in principle, each such solution can be expressed by theta functions on some algebraic curve. In the rather instructive simple example of a curve in the 2-sphere (which we will not persue in this paper) the spectral parameter scales the speed of the curve and at the same time scales the (geodesic) curvature inverse proportional. The lowest type curves give the geodesics (spectral curve is the Riemann sphere) and elastic curves (spectral curve is elliptic).

Second author was partially supported by NSF grant DMS-9205293, the SFB 288 at Technische Universität Berlin and the Graduierten Kolleg at Humboldt Universität Berlin. 
We would like to thank Fran Burstall, Ottmar Loos and Ulrich Pinkall for stimulating discussions during the preparation of this paper. Finally, the second author would like to thank the members of the SFB 288 at TU-Berlin for their interest and hospitality.

\section{Curvature isotropic immersions}

Let $N=G / K$ be a semisimple symmetric space endowed with the Killing metric, $M$ a manifold of dimension $m$, and $f: M \rightarrow N$ a smooth map. Since most of our considerations are local, we assume $M$ to be contractible unless otherwise stated.

Definition. $f$ is called curvature isotropic or a curved flat, if we have

$$
f^{*} R^{N}=0
$$

as a 2 -form on $M$ with values in the bundle $\operatorname{End}\left(f^{*} T N\right)$. Here $R^{N}$ denotes the curvature tensor of $N$.

Moreover, if $f$ is an immersion which induces a nondegenerate (not necessarily positive definite) metric on $\mathrm{M}$, we call $f$ a regular curved flat.

We give a Lie algebraic formulation of this definition, which will also explain the term curved flat. Let

$$
\mathfrak{g}=\mathfrak{k}+\mathfrak{p} .
$$

be the symmetric decomposition of $G / K$. Let $F: M \rightarrow G$ be a lift of $f$ so that $\pi \circ F=f$ where $\pi: G \rightarrow G / K$, $\pi(g)=[g]$, is the coset projection. We call such a lift a framing of $f$. Then

$$
F^{-1} \mathrm{~d} F=A=A_{0}+A_{1},
$$

where $A$ is a 1 -form on $M$ with values in $\mathfrak{g}$, and $A_{0}$ resp. $A_{1}$ denote the $\mathfrak{k}$ resp. p parts of $A$. $A$ satisfies the Maurer-Cartan equation or integrability condition

$$
\mathrm{d} A+\frac{1}{2}[A \wedge A]=0
$$

where

$$
[A \wedge B](X, Y)=[A(X), B(Y)]-[A(Y), B(X)] .
$$

The $\mathfrak{p}$-space is closed under the triple Lie bracket and canonically identified with the tangent space of $N$ at the origin $[e]=K$. Then, if $f(p)=[e], F(p)=e$, and $X \in T_{p} M$, we obtain

$$
\begin{aligned}
f_{*}(X) & =\pi_{*} F_{*}(X)=\pi_{*}(F A(X)) \\
& =\pi_{*}\left(A_{0}(X)+A_{1}(X)\right)=\pi_{*} A_{1}(X) \\
& =A_{1}(X),
\end{aligned}
$$

and

$$
\begin{aligned}
f^{*} R^{N}(X, Y) & =-\frac{1}{2}\left[\left[A_{1}(X), A_{1}(Y)\right], \ldots\right] \\
& =-\frac{1}{2}\left[\left[A_{1} \wedge A_{1}\right](X, Y), \ldots\right]
\end{aligned}
$$

Lemma 1. The subspace $\mathfrak{a}:=A_{1}\left(T_{p} M\right)$ is abelian:

$$
\left[A_{1} \wedge A_{1}\right]=0 .
$$

Proof. The assumption implies

$$
[[\mathfrak{a}, \mathfrak{a}], \mathfrak{p}]=0
$$

Note that

$$
\mathfrak{g}_{1}:=[\mathfrak{p}, \mathfrak{p}]+\mathfrak{p} \text { is an ideal of } \mathfrak{g} \text {. }
$$


Since $\mathfrak{g}$ is semisimple, so is $\mathfrak{g}_{1}$, and by 2.4 , $[\mathfrak{a}, \mathfrak{a}]$ is in the center of $\mathfrak{g}_{1}$. Hence $[\mathfrak{a}, \mathfrak{a}]=0$.

Conversely, a $\mathfrak{g}$-valued 1 -form $A$ that satisfies the integrability condition (2.2) together with (2.3) gives rise to a curvature isotropic map f.

The Lemma shows that a curved flat is at each point tangent to a flat totally geodesic submanifold of $N$.

In view of (2.3) the $\mathfrak{k}$ - and $\mathfrak{p}$-part of $(2.2)$ reduce to

$$
\begin{aligned}
\mathrm{d} A_{0}+\frac{1}{2}\left[A_{0} \wedge A_{0}\right] & =0 \\
\mathrm{~d} A_{1}+\left[A_{0} \wedge A_{1}\right] & =0
\end{aligned}
$$

Note that $A_{0}: T M \rightarrow \mathfrak{k}$ is the canonical connection of the principal $K$-bundle $f^{*}(G) \rightarrow M$, and this is flat by equation (2.5).

\section{EXAMPLES}

Example 1. Any curve in a symmetric space, in particular in the rank 1 space $S^{2}$ is curvature isotropic. This rather trivial example nicely displays several features of the general theory in a very explicit form. We shall come back to this elsewhere.

Example 2. Let $M^{m}$ be of constant curvature $c>0$, and let

$$
\phi: M^{m} \rightarrow S:=S^{n+1}(c) \subset \mathbb{R}^{n+2}
$$

be an isometric immersion into the sphere of equal curvature $c$. The Gauss map of $\phi$

$$
f=\gamma_{\phi}: M \rightarrow G_{m+1}\left(\mathbb{R}^{n+2}\right)=\frac{\mathbf{O}(n+2)}{\mathbf{O}(m+1) \times \mathbf{O}(n+1-m)}
$$

assigns to each point of $\mathrm{M}$ the orthogonal complement of its normal space in the sphere $S$ considered as linear subspace of $\mathbb{R}^{n+2}$. If $\phi$ has flat normal bundle $V^{\perp}$, we claim that

$$
\gamma_{\phi} \text { is curvature isotropic. }
$$

For $p \in M$ we define

$$
V_{p}=\gamma_{\phi}(p)=\mathbb{R} \phi(p) \oplus \phi_{*}\left(T_{p} M\right)
$$

Then

$$
M \times \mathbb{R}^{n+2}=V \oplus V^{\perp}
$$

The bundle $V^{\perp}$ is flat by assumption, but $V$ is flat, too. To see this, we consider the second fundamental form $\beta$ of $V$ :

$$
\beta: T M \times V \rightarrow V^{\perp}, \beta(X, s)=\left(D_{X} s\right)^{V^{\perp}}
$$

Then

$$
<R^{V}(X, Y) s, t>=<\beta(X, s), \beta(Y, t)>-<\beta(Y, s), \beta(X, t)>
$$

But for $X, Y \in T M$

$$
\begin{aligned}
\beta(X, \phi) & =0 \\
\beta\left(X, \phi_{*} Y\right) & =I I_{\phi}(X, Y),
\end{aligned}
$$

where $I I_{\phi}$ denotes the second fundamental form of $\phi$. Since $M$ and $S$ have the same curvature, this together with the Gauss equation implies, that the right-hand side of (3.1) is zero. 
Now $V$ and $V^{\perp}$ are the pull-backs of the canonical bundle $\nu$ and its orthogonal complement $\nu^{\perp}$ over $G_{m+1}\left(\mathbb{R}^{n+2}\right)$. The tangent bundle of the Grassmannian can be identified with $\operatorname{Hom}\left(\nu^{\perp}, \nu\right)$ and for tangent vector fields $X, Y, Z$ the curvature tensor of $G_{m+1}\left(\mathbb{R}^{n+2}\right)$ is given by

$$
(R(X, Y) Z)(s)=R^{\nu}(X, Y) Z(s)-Z\left(R^{\nu^{\perp}}(X, Y) s\right), s \in \Gamma\left(\nu^{\perp}\right) .
$$

The flatness of $V$ and $V^{\perp}$ therefore implies that $\gamma_{\phi}$ is curvature isotropic.

If we canonically identify

$$
T_{\gamma_{\phi}} G_{m+1}\left(\mathbb{R}^{n+2}\right)=\operatorname{Hom}\left(V_{p}, V_{p}^{\perp}\right),
$$

then

$$
d_{p} \gamma_{\phi}(X)(s)=\beta(X, s)
$$

If we assume that the kernel of the second fundamental form of $\phi$ is trivial or, equivalently, that $f=\gamma_{\phi}$ is immersive, then we see from (3.2), (3.3) that $\phi(p)$ is characterized uniquely up to sign as a unit vector in the kernel of $d_{p} \gamma_{\phi}\left(T_{p} M\right)$. This allows the reconstruction of $\phi$ from the associated curved flat.

This example can be worked out similarly for isometric immersions between hyperbolic spaces of equal curvature. The ambient space then is the Lorentzian $\mathbb{R}_{1}^{n+2}$, and the Gauss map is a space-like map into the $(m+1)$-planes of index 1 .

Conversely, we can construct isometric immersions between space forms of equal curvature from curved flats into the appropriate Grassmannians: if

$$
f: M^{m} \rightarrow G_{m+1}\left(\mathbb{R}^{2 m+1}\right)
$$

or

$$
f: M^{m} \rightarrow G_{m+1}\left(\mathbb{R}_{1}^{2 m+1}\right)
$$

is a space-like regular curved flat, then $A_{1}\left(T_{p} M\right)$ is $m$-dimensional for all $p$. Moreover, if it is a Cartan subalgebra (see Remark 1 below), then there exists a gauge $H: M \rightarrow K$, where $K$ is either $\mathbf{O}(m+1) \times \mathbf{O}(m)$ or $\mathbf{O}(1, m) \times \mathbf{O}(m)$, so that the gauged frame $\tilde{F}=F H$ has

$$
\tilde{F}^{-1} \mathrm{~d} \tilde{F}=\left(\begin{array}{c|c|ccc} 
& \mp \sigma^{T} & & & \\
\hline & & -\beta_{1} & & \\
\sigma & \omega & & \ddots & \\
& & & & -\beta_{m} \\
\hline & * & & \eta &
\end{array}\right) .
$$

This follows from Remark 1 below together with the fact that the p-part of the above matrix, $\tilde{A}_{1}$, represents a normal form of space-like Cartan subalgebras in $\mathfrak{p}$. Thus we see that $\tilde{F}(p) e_{0} \in \operatorname{ker} \tilde{A}_{1}\left(T_{p} M\right)$. Now consider the map $\phi=\tilde{F} e_{0}$ which has its image in $S^{2 m}$ or $H^{2 m}$. If $\phi$ is immersive then $\tilde{F}$ is an adapted frame for $\phi$ and (3.1) implies that $\phi$ is an isometric immersion between space forms of equal curvature and flat normal bundle.

Example 3. Let $M^{m}$ be of constant curvature $\left.c \in\right] 0,1[$, and let

$$
\phi_{0}: M^{m} \rightarrow S^{n}(1)
$$

be an isometric immersion with flat normal bundle. Note that the latter condition is automatic if $n=2 m-1$, see [6]. We embed $S^{n}(1)$ as an umbilic hypersurface into a sphere $S=S^{n+1}(c) \subset \mathbb{R}^{n+2}$ of curvature $c$, and obtain an isometric immersion $\phi$ between constant curvature spaces of equal curvatures as in the the preceeding example.

This construction is not limited to the above curvature relations. Consider an $m$-dimensional space form $\mathrm{M}$ of curvature $c$ isometrically immersed with flat normal bundle into an $n$-dimensional space form of curvature $\tilde{c} \neq c \neq 0$. The latter can always be realized as an umbilic hypersurface of a hyperquadric $S$ again of curvature $c$ in some Euclidean or pseudo-Euclidean $(n+2)$-space. This produces examples of curvature isotropic maps into the Grassmanianns given in the following list. 


\begin{tabular}{|c|c|c|c|}
\hline curvatures & $\mathrm{S}$ & ambient space & Grassmannian \\
\hline$\tilde{c}>c>0$ & $S^{n+1}(c)$ & $\mathbb{R}^{n+2}$ & $\frac{\mathbf{O}(n+2)}{\mathbf{O}(m+1) \times \mathbf{O}(n+1-m)}$ \\
\hline$\tilde{c}<c>0$ & $S_{1}^{n+1}(c)$ & $\mathbb{R}_{1}^{n+2}$ & $\frac{\mathbf{O}(1, n+1)}{\mathbf{O}(1+m) \times \mathbf{O}(1, n-m)}$ \\
\hline$\tilde{c}>c<0$ & $H^{n+1}(c)$ & $\mathbb{R}_{1}^{n+2}$ & $\frac{\mathbf{O}(1, n+1)}{\mathbf{O}(1, m) \times \mathbf{O}(n+1-m)}$ \\
\hline$\tilde{c}<c<0$ & $H_{1}^{n+1}(c)$ & $\mathbb{R}_{2}^{n+2}$ & $\frac{\mathbf{O}(2, n)}{\mathbf{O}(1, m) \times \mathbf{O}(1, n-m)}$ \\
\hline
\end{tabular}

The isometric immersions between space forms of equal curvature that arise in the above manner carry a distinguished umbilic normal field, namely that of the ambient umbilic hypersurface of $S$. Conversely, consider an isometric immersion $\phi$ with flat normal bundle and immersive Gauss map between space forms of equal curvature. Assume that we are in the critical codimension $n=2 m-1$. Then we can show the existence of a distinguished umbilic normal field $\xi$, and prove that the constancy of $|\xi|$ is necessary and sufficient for $\phi$ to factor through an umbilic hypersurface of $S$.

A different approach for the construction of isometric immersions of space forms of distinct curvatures is presented in 沟.

Example 4. A curved 2-flat in the Grassmannian $G_{3}^{+}\left(\mathbb{R}_{1}^{5}\right)$ of space-like 3-planes in Lorentzian 5-space has a lift

$$
F: M^{2} \rightarrow \mathbf{O}(1,4) .
$$

The Lorentz group acts on the sphere $S_{1}^{4}:=\left\{v \in \mathbb{R}_{1}^{5} \mid\langle v, v\rangle=1\right\}$, and an appropriate orbit of $F$ gives a special Ribeaucour sphere congruence in $S^{3}$ enveloping a pair of isothermic surfaces. See [2], p.4.

\section{Geometry of CURVED Flats}

We have seen that a curved flat $f: M \rightarrow N$ is at each point tangential to flats in the symmetric space $N$. As we shall see below this implies (under certain non-degeneracy assumptions) that a curved flat is intrinsically flat (where the metric on $N$ is always the one induced by the Killing form of $\mathfrak{g}$ ). In this sense curved flats can be regarded as analogues of developable surfaces in Euclidean space. Moreover, if the curved flat is the Gauss map of an isometric immersion then the developing isometry induces principal curvature coordinates on $M$. In this situation the flat metric has been used to show certain non-existence results for isometric immersions [7.

Theorem 2. Let $f: M \rightarrow N$ be a regular curved flat whose tangent spaces are all conjugate under the action of the connected component of the isotropy group of $N$. Then $M$ with the induced metric $f^{*} g_{N}$ is (intrinsically) flat.

Remark 1. The transitivity assumption of the Theorem will hold provided that all tangent spaces of the curved flat are Cartan subalgebras: this follows from the fact that the connected components of the space of all Cartan subalgebras in $\mathfrak{p}$ are precisely the orbits under (the connected component of) the isotropy group $K_{0}$. Recall that a subspace $\mathfrak{a}$ of $\mathfrak{p}$ is a Cartan subalgebra if $\mathfrak{a}$ is maximal abelian and consists of semi-simple elements. All Cartan subalgebras have the same dimension [1], say $r$, which defines the rank of the (pseudo-Riemannian) symmetric space $G / K$. Now consider

$$
\Sigma=\left\{\mathfrak{a} \subset \mathfrak{p}^{\mathbb{C}} ; \mathfrak{a} \text { abelian and } \operatorname{dim}_{\mathbb{C}} \mathfrak{a}=r\right\} \subset G_{r}\left(\mathfrak{p}^{\mathbb{C}}\right)
$$

which is a subvariety (defined over $\mathbb{R}$ ) of the Grassmanian of $r$-planes in $\mathfrak{p}^{\mathbb{C}}$. The set of all Cartan subalgebras

$$
\widetilde{\Sigma}=\{\mathfrak{a} \in \Sigma ; \mathfrak{a} \text { Cartan }\}
$$

is open in $\Sigma$ since it is easy to show that a maximal abelian $\mathfrak{a}$ is Cartan iff the Killing form is non-degenerate on $\mathfrak{a}$ and the set of maximal abelian $\mathfrak{a} \in \Sigma$ is open in $\Sigma$. Moreover, $K^{\mathbb{C}}$ acts transitively on $\widetilde{\Sigma}$ with stabilizer 
$N\left(\mathfrak{a}_{0}\right)=\left\{k \in K^{\mathbb{C}} ; \operatorname{Ad} K^{\mathbb{C}}\left(\mathfrak{a}_{0}\right)=\mathfrak{a}_{0}\right\}, \mathfrak{a}_{0} \in \widetilde{\Sigma}$, so that all points in $\widetilde{\Sigma} \subset \Sigma$ are smooth and $\widetilde{\Sigma}=K^{\mathbb{C}} / N\left(\mathfrak{a}_{0}\right)$. Let

$$
\Sigma(\mathbb{R})=\left\{\mathfrak{a} \subset \mathfrak{p} ; \mathfrak{a} \text { abelian and } \operatorname{dim}_{\mathbb{R}} \mathfrak{a}=r\right\} \subset G_{r}(\mathfrak{p})
$$

be the $\mathbb{R}$-points of the variety $\Sigma$. Then the set of real Cartan subalgebras in $\mathfrak{p}$ is

$$
\widetilde{\Sigma}_{\mathbb{R}}=\widetilde{\Sigma} \cap \Sigma(\mathbb{R})
$$

and as above $\widetilde{\Sigma}_{\mathbb{R}}$ is open in $\Sigma(\mathbb{R})$. Moreover, 10$], \widetilde{\Sigma}_{\mathbb{R}}$ is a smooth real manifold of $\operatorname{dim}_{\mathbb{R}} \widetilde{\Sigma}_{\mathbb{R}}=\operatorname{dim}_{\mathbb{C}} \widetilde{\Sigma}$ with only finitely many connected components. To see that each $K_{0}$-orbit $\mathcal{O}$ is a connected component of $\widetilde{\Sigma}_{\mathbb{R}}$ it suffices to show that $\mathcal{O}$ is open in $\widetilde{\Sigma}_{\mathbb{R}}$. Since $\mathcal{O}=K_{0} / N\left(\mathfrak{a}_{0}\right)$ where $N\left(\mathfrak{a}_{0}\right)$ is the normalizer of some $\mathfrak{a}_{0} \in \widetilde{\Sigma}_{\mathbb{R}}$ we obtain

$$
\operatorname{dim}_{\mathbb{R}} \mathcal{O}=\operatorname{dim}_{\mathbb{C}} \widetilde{\Sigma}=\operatorname{dim}_{\mathbb{R}} \widetilde{\Sigma}_{\mathbb{R}}
$$

which shows that $\mathcal{O}$ is open in $\widetilde{\Sigma}_{\mathbb{R}}$.

In the above discussion we saw that being a Cartan subalgebra is an open condition inside rank dimensional abelian subspaces. When we integrate the curved flat equations in Section 6 we can always assure this locally by choosing the right initial conditions for the Lax flows.

Of course, for Riemannian symmetric spaces $\mathrm{N}$ it suffices to have $\operatorname{dim} M=\operatorname{rank} N$ for the tangent spaces of a regular curved flat $f: M \rightarrow N$ to be Cartan.

Proof of the Theorem. We argue locally at $p_{0} \in M$, and may hence assume the existence of a framing $F: M \rightarrow G$ of $f$, such that as in (2.1)

$$
\begin{aligned}
F^{-1} \mathrm{~d} F & =A=A_{0}+A_{1}, \\
F\left(p_{0}\right) & =e \in G .
\end{aligned}
$$

Then

$$
\mathfrak{a}_{p}:=f_{*}\left(T_{p} M\right)=A_{1}\left(T_{p} M\right) \subset \mathfrak{p}
$$

is abelian by Lemma 1 . We change $F$ by a gauge transformation using the transitivity assumption. Let $H: M \rightarrow K$ be such that $\operatorname{Ad}_{H(p)}\left(\mathfrak{a}_{p}\right)=\mathfrak{a}_{p_{0}}$, and put $\tilde{F}:=F H$. Then taking the $\mathfrak{p}$-part of the Maurer-Cartan equation (2.2) for the corresponding

$$
\tilde{A}_{1}=\operatorname{Ad}(H) A_{1}: T M \rightarrow \mathfrak{a}_{p_{0}}
$$

we find

$$
\mathrm{d} \tilde{A}_{1}+2\left[\tilde{A}_{0} \wedge \tilde{A}_{1}\right]=0 .
$$

Clearly d $\tilde{A}_{1}$ has values in $\mathfrak{a}_{p_{0}}$, while

$$
<\left[\mathfrak{k}, \mathfrak{a}_{p_{0}}\right], \mathfrak{a}_{p_{0}}>=<\mathfrak{k},\left[\mathfrak{a}_{p_{0}}, \mathfrak{a}_{p_{0}}\right]>=0
$$

implies that $\left[\tilde{A}_{0} \wedge \tilde{A}_{1}\right]$ has values in $\mathfrak{a}_{p_{0}}^{\perp}$. Since $f$ was assumed to be regular $\mathfrak{a}_{p_{0}}$ and $\mathfrak{a}_{p_{0}}^{\perp}$ are complementary subspaces.

Therefore

$$
\mathrm{d} \tilde{A}_{1}=0
$$

and so $\tilde{A}_{1}=\mathrm{d} \psi$ for some local diffeomorphism $\psi: M \rightarrow \mathfrak{a}_{p_{0}}$. Finally

$$
\begin{aligned}
<f_{*} X, f_{*} Y>= & <\pi_{*} \tilde{F}_{*} X, \pi_{*} \tilde{F}_{*} Y> \\
= & <\tilde{A}_{1}(X), \tilde{A}_{1}(Y)>_{\mathfrak{a}_{p_{0}}} \\
= & <d \psi(X), d \psi(Y)>\operatorname{a}_{p_{0}}
\end{aligned}
$$

shows that the metric is flat. 
Remark 2. Let $f: M \rightarrow N$ be a regular curved flat such that all its tangent spaces are Cartan subalgebras. As in the proof let $\mathfrak{a}=f_{*}\left(T_{p_{0}} M\right)$ be a fixed Cartan subspace in $\mathfrak{p}$ and $\psi: M \rightarrow \mathfrak{a}$ the developing isometry. If $\alpha_{1}, \ldots, \alpha_{m}$ are a set of simple roots on $\mathfrak{a}$ then $\alpha_{i} \circ \psi$ give canonical coordinates on $M$. If the curved flat arises from an isometric immersion between space forms like at the end of Example 2 then one can easily check that these coordinates are the principal curvature coordinates for the immersion. This provides a rather nice geometric interpretation of the developing isometry of a curved flat.

\section{The SPECTRAL PARAMETER}

In this section we reformulate the curved flat equations (2.2), (2.3) as an integrability condition involving an additional - spectral - parameter. If the curved flat happens to be the Gauss map of an isometric immersion (Example 2) this parameter scales the second fundamental form of the immersion and thus gives a non-trivial deformation. The existence of such a non-trivial deformation is indicative that curved flats are solutions to a certain integrable system. We will discuss this aspect in more detail in Section 6 .

Let $A_{0}$ and $A_{1}$ be $\mathfrak{k}$ and $\mathfrak{p}$-valued 1-forms and consider the 1-parameter family (loop) of $\mathfrak{g}$-valued 1-forms

$$
A^{\mu}:=A_{0}+\mu A_{1}
$$

for real $\mu \neq 0$. Then we have

Lemma 3. The following are equivalent statements:

i. $A_{0}$ and $A_{1}$ satisfy the curved flat equations (2.2) and (2.3);

ii. $A_{0}$ and $\mu A_{1}$ satisfy the curved flat equations (2.2) and (2.3) for some $\mu \neq 0$;

iii. $A^{\mu}$ solves the Maurer-Cartan equation

$$
\mathrm{d} A^{\mu}+\frac{1}{2}\left[A^{\mu} \wedge A^{\mu}\right]=0
$$

identical in $\mu$.

The proof follows immediately by comparing coefficients at powers of $\mu$. Thus we see that curved flats come in 1-parameter families and that their integrability equations can be rewritten as a zero-curvature condition involving and auxiliary parameter.

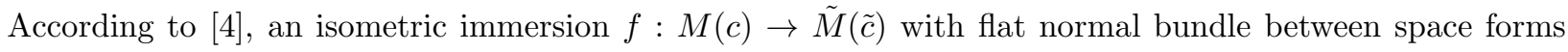
of distinct curvatures also comes in a 1-parameter family - let us call this parameter $\lambda$. As explained in Example 3, their Gauss map is a curved flat, which by the above also embeds in a 1-parameter family. It can be shown that the two families correspond where the relation between the two parameters is given by

$$
\mu=\frac{-\sqrt{c}}{2 \sqrt{1-c}}\left(\lambda-\lambda^{-1}\right) .
$$

\section{Integration of the Curved Flat Equations}

Using the reformulation of the curved flat equations (Lemma 3) as a loop of flat 1-forms we will now apply the finite type integration scheme to construct solutions in terms of a hierarchy of finite dimensional commuting ODE in Lax form. Let $N=G / K$ be a semisimple symmetric space,

$$
\mathfrak{g}=\mathfrak{k}+\mathfrak{p}
$$

the symmetric decomposition of $G / K$, and denote by $\sigma: \mathfrak{g} \rightarrow \mathfrak{g}$ the corresponding involution. We put

$$
\Lambda \mathfrak{g}_{\sigma}:=\left\{\xi: \mathbb{R}^{*} \rightarrow \mathfrak{g} \text { Laurent polynomial in } \mu \mid \sigma \xi(\mu)=\xi(-\mu)\right\}
$$

and split this as

$$
\Lambda \mathfrak{g}_{\sigma}=\Lambda^{+} \mathfrak{g}_{\sigma} \oplus \Lambda_{*}^{-} \mathfrak{g}_{\sigma} .
$$


Here $\Lambda^{+} \mathfrak{g}_{\sigma}$ denotes the polynomial loops in $\mu$, while those in $\Lambda_{*}^{-} \mathfrak{g}_{\sigma}$ are polynomials in $\mu^{-1}$ that vanish at infinity. Let $\pi_{ \pm}$denote the corresponding projections. Then

$$
R:=\frac{1}{2}\left(\pi_{+}-\pi_{-}\right): \Lambda \mathfrak{g}_{\sigma} \rightarrow \Lambda \mathfrak{g}_{\sigma}
$$

defines an R-matrix, and we can apply the results of [5], Section 2, and [3], Section 3.

Let $d>0$ be odd, and let $V$ be an $\operatorname{Ad} G$-equivariant polynomial vector field on $\mathfrak{g}$. We decompose this into its homogeneous parts of degrees $r_{1}, \ldots, r_{m}$,

$$
V=V^{(1)}+\ldots+V^{(m)}
$$

and define for $\xi \in \Lambda \mathfrak{g}_{\sigma}$

$$
\tilde{V}(\xi)(\mu):=\sum_{i=1}^{m}(-1)^{r_{i}+1} \mu^{1-d r_{i}} V^{(i)}(\xi(\mu))
$$

If $V$ is $(-\sigma)$-equivariant

$$
-\sigma V(\xi)=V(-\sigma \xi)
$$

then $\tilde{V}$ is ad-equivariant on $\Lambda \mathfrak{g}_{\sigma}$, and induces a vector field

$$
X_{V}(\xi)=\left[\xi,\left(R+\frac{1}{2}\right) \tilde{V}(\xi)\right]
$$

on $\Lambda \mathfrak{g}_{\sigma}$, which is in fact tangential to the finite dimensional subspace

$$
\Lambda_{d}:=\left\{\xi \in \Lambda^{+} \mathfrak{g}_{\sigma} \mid \xi=\sum_{k=0}^{d} \mu^{k} \xi_{k}\right\} .
$$

Any $k$ such vector fields $C^{\infty}$-commute. Natural examples are given by the gradients $\operatorname{grad} v=V$ of $\operatorname{Ad} G$ and $(-\sigma)$-invariant polynomials $v$ (with respect to an $\mathrm{Ad} G$-invariant inner product on $\mathfrak{g}$ ). If $\mathrm{N}$ is of rank $k$, then the existence of $k$ independent such functions is guaranteed, provided $\mathrm{N}$ has the surjection property, see [3] Section 8, which is the case for all classical symmetric spaces and group manifolds.

We now are in the position to state the basic integration theorem for curved flats:

Theorem 4. Given $\operatorname{Ad} G$-and $(-\sigma)$-equivariant polynomial vector fields $V_{1}, \ldots, V_{k}$ on $\mathfrak{g}$, and $\xi_{0} \in \Lambda_{d}$, for some odd $d>0$, the system

$$
\frac{\partial \xi}{\partial x_{j}}=\left[\xi,\left(R+\frac{1}{2}\right) \tilde{V}_{j}(\xi)\right]
$$

has a unique local solution $\xi: \mathbb{R}^{k} \supset U \rightarrow \Lambda_{d}$ with $\xi(0)=\xi_{0}$. For this

$$
A^{\mu}=\sum_{j}\left(R+\frac{1}{2}\right) \tilde{V}_{j}(\xi) d x^{j}
$$

is of the form (5.1). It satisfies (2.2), (2.3) for all $\mu$, and therefore induces a curvature isotropic map $f: \mathbb{R}^{k} \supset U \rightarrow N$.

Remark 3. If the rank of $G / K$ is $k$ then we can choose the initial condition $\xi_{0}$ in such a way that $\left(R+\frac{1}{2}\right) \tilde{V}_{j}\left(\xi_{0}\right)$, $j=1, \ldots, k$ span a Cartan subalgebra in $\mathfrak{p}$. But this is an open condition inside $k$-dimensional abelian subspaces and thus each tangent space of the resulting curved flat will be a Cartan subalgebra for $p \in U$ (c.f. Remark 1).

Remark 4. If $G / K$ is a Grassmannian - which is the case for the Gauss maps of isometric immersions the vector fields $V_{i}$ are simply given by even powers of off-blockdiagonal matrices $\xi^{2 i}$. 


\section{REFERENCES}

[1] M. Berger, Les espaces symétriques non compacts, Ann. Ec. Norm. Sup. 74 (1957), 85-177.

[2] F. Burstall, U. Hertrich-Jeromin, F. Pedit, U. Pinkall, Curved flats and isothermic surfaces, SFB 288 Preprint No. 132 (1994)

[3] F.E. Burstall, D. Ferus, F. Pedit, U. Pinkall, Harmonic tori in symmetric spaces and commuting Hamiltonian systems on loop algebras, Ann. of Math. 138 (1993),173-212.

[4] D. Ferus, F. Pedit, Isometric immersions of space forms and soliton theory, SFB 288 Preprint No. 154 (1995).

[5] D. Ferus, F. Pedit, U. Pinkall, I. Sterling, Minimal tori in $S^{4}$, J. Reine Angew. Math. 429 (1992), 1-47.

[6] J.D. Moore, Isometric immersions of space forms into space forms, Pacific J. Math. 40 (1972),157-166.

[7] F. Pedit, A non-immersion theorem for space forms, Comment. Math. Helvetici 63 (1988), 672-674.

[8] U. Hertrich-Jeromin, Conformally flat hypersurfaces and integrable systems, Thesis, TU-Berlin (1994).

[9] U. Hertrich-Jeromin, On conformally flat hypersurfaces, curved flats and cyclic systems, in preparation.

[10] H. Whitney, Elementary structure of real algebraic varieties, Ann. of Math. 66 (1957), 545-556.

Fachbereich Mathematik, MA 8-3, Technische Universität Berlin, Strasse des 17. Juni 136, 10622 Berlin, Germany

E-mail address, Dirk Ferus: ferus@sfb288.math.tu-berlin.de

Department of Mathematics, University of Massachusetts, Amherst, MA 01003

E-mail address, Franz Pedit: franz@gang.umass.edu 